\title{
Fertilización mineral y orgánica en el cultivo intercalado maíz-frijol asociados y haba en un Andosol del Estado de México
}

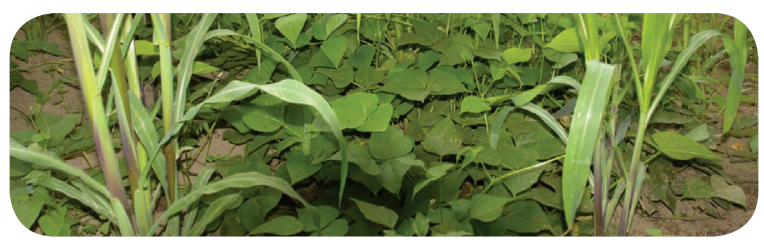

\author{
Laura García Tapia ${ }^{1}$ \\ Ildefonso Ronquillo Cedillo ${ }^{2}$
}

Recepción: 28/05/2018 Aceptación: 11/08/2018

\section{Resumen}

La producción agrícola en San José del Rincón, Estado de México, como en el país en general, presenta problemas ambientales, económicos y sociales. La implementación de tecnologías no adaptadas a las condiciones locales ha reducido la productividad de los sistemas campesinos. El objetivo fue evaluar la productividad, eficiencia relativa de la tierra (ERT) e ingreso neto familiar del cultivo intercalado en franjas de maíz-frijol de guía asociados y haba en un Andosol, con fertilización mineral y orgánica.

La investigación se realizó en la parcela de un productor en el ciclo primavera-verano de temporal del 2014. El diseño experimental fue en parcelas divididas con dos arreglos topológicos (AT) como parcela grande (un surco de maíz-frijol de guía asociados, intercalados con un surco de haba (1x1), y dos surcos de maíz-frijol de guía asociados, intercalados con dos surcos de haba (2x2)) y nueve tratamientos de parcela chica, seleccionados con una matriz de un factor a la vez (matriz baconiana).

Los factores fueron: fertilización, densidad de población y variedad de maíz. Se adicionaron tres tratamientos de cultivo simple para calcular la eficiencia relativa de la tierra. Los resultados indicaron que el cultivo intercalado $1 \times 1$ con maíz azul y el 2 x2 con maíz rosado; fertilización 80-80-00 con 3tha ${ }^{-1}$ de composta, obtuvo una eficiencia relativa de la tierra de 1.35 y 1.40 respectivamente, con un ingreso equivalente a 1.8 y 1.24 salarios mínimos por día. Se concluye que el cultivo intercalado de maíz-frijol asociados y haba aumenta el rendimiento y el ingreso sobre el cultivo simple y es opción para los campesinos.

Palabras clave: Policultivos, productividad agrícola, eficiencia relativa de la tierra, ingresos netos.

\begin{abstract}
Agricultural production in San José del Rincón, State of Mexico, as in the country in general, presents environmental, economic and social problems. The implementation of technologies not adapted to local conditions has reduced the productivity of peasant systems. The objective was to evaluate the productivity, Relative Efficiency of the Land (REL) and family net income of the intercropping in fringes of corn-associated guide bean and beans in an Andosol, with mineral and organic fertilization.

The research was conducted on a producer's plot in the spring-summer cycle of 2014. The experimental design was divided into plots with two topological arrangements (TA) as a large plot (a groove of corn-bean associated guide, interleaved with a bean groove (1x1), and two groves of associated corn-bean guide, interspersed with two bean grooves $(2 \times 2))$ and nine small-plot treatments, selected with a one-factor matrix at a time (Baconian matrix) ).

The factors were: fertilization, population density and variety of corn. Three simple culture treatments were added to calculate the relative efficiency of the land. The results indicated that the intercropping $1 \times 1$ with blue corn and the $2 \times 2$ with pink corn; fertilization 80-80-00 with 3tha- 1 of compost, obtained a relative efficiency of the earth of 1.35 and 1.40 respectively, with an income equivalent to 1.8 and 1.24 minimum wages per day. It is concluded that the intercropping of maize-beans associated with bean increases the yield and income on the simple crop and is an option for the farmers.
\end{abstract}

Key words: Polycultures, agricultural productivity, relative land efficiency, net income.

1. Maestra en Ciencias, Investigadora independiente; e-mail: laura.garcia@colpos.mx

2. Maestro en Ciencias, Profesor-investigador, Universidad Intercultural del Estado de México; e-mail: ildefonso.ronquillo@uiem.edu.mx 


\section{Introducción}

La producción agrícola en la región de San José del Rincón, Estado de México, presenta diversos problemas ambientales, económicos y sociales, como erosión hídrica moderada causada por actividades agrícolas que han disminuido la fertilidad del suelo en un $40 \%$ (SEMARNAT-COLPOS, 2002). Los suelos predominantes son Andosoles, caracterizados por su fácil degradación cuando la vegetación es removida; y por fijar fósforo cuando presentan propiedades ándicas (IUSS Grupo de Trabajo WRB, 2014), por lo que disminuye su disponibilidad para las plantas (García et al., 2012). El problema se agrava cuando se cultiva papa con elevados requerimientos nutricionales, generando la disminución de la fertilidad y ocasionando abandono de tierras o cambio de cultivo con bajos rendimientos (Núñez et al., 2003).

Los campesinos poseen menos de cinco hectáreas, dispersas en pequeños predios agrícolas en laderas con pendientes de $20 \%$ donde cultivan blanco, azul y rosado en cultivo simple (SIAP, 2013). En menor porcentaje también se utiliza el sistema milpa, que incluye especies como calabaza, haba y frijol para obtener una mayor diversidad de alimentos para autoconsumo (Mendieta et al., 2013).

La mayoría de las tierras son de temporal (de 24,910.5ha cultivadas; solo 5.2ha usan riego); 20,550ha son sembradas con maíz; 2,400ha con avena forrajera; 1250ha con triticale; 610ha de papa y 100.5ha con haba, nopal, zanahoria y jitomate y algunas praderas (SIAP, 2017). Debido a que la producción es incierta por diversos factores adversos, la agricultura está siendo desplazada como actividad principal, al no representar un sustento sólido para las familias locales que emigran a las ciudades en busca de trabajo (Mendieta et al., 2013). En este entorno, agroecosistemas tradicionales, tales como la milpa, son una base sólida para encontrar soluciones a la problemática no solo de la degradación de los suelos, sino también una solución económica para los campesinos.

Investigaciones agronómicas en Andosoles de la Sierra Tarasca, Michoacán (México), indican que el rendimiento de grano de maíz se puede incrementar con una fertilización orgánica-mineral (Guerrero, 1987), con una densidad de población adecuada y mediante el arreglo topológico de maíz y frijol bajo el sistema de cultivo intercalado en franjas; sin incrementar la dosis de fertilización respecto al cultivo simple de maíz (Albino-Garduño et al., 2015). No obstante, los Andosoles de esta región son más desarrollados y su capacidad de fijación de fosfatos es más alta (Alcalá et al., 2001), por lo que la trasferencia de tecnología a la zona de estudio no se puede realizar sin un estudio previo de sus propiedades; y sin realizar experimentos sobre la productividad y eficiencia del sistema. 
El objetivo de la presente investigación fue evaluar la viabilidad del cultivo maíz-frijol de guía asociados intercalado en franjas con haba con fertilización mineral y orgánica.

\section{Materiales y Métodos}

El estudio se realizó en el ciclo primavera-verano de temporal del año 2013, en la comunidad de San Juan Palo Seco, San José del Rincón, Estado de México, en la región noroeste de dicho Estado. El clima de la región es templado subhúmedo, con lluvias en verano de 800 a $1200 \mathrm{~mm}$, a una altura de $2838 \mathrm{msnm}$ y temperatura de 10 a $12^{\circ} \mathrm{C}$.

Para caracterizar el suelo, se excavó un perfil colectando muestras de cada horizonte, y se describieron morfológicamente con base en Cuanalo (1990). En el laboratorio de sue- los del Colegio de Postgraduados campus Montecillo, se realizaron los análisis físicos y químicos (Cuadro 1) con base en el manual de Van Reeuwijk (1999): contenido de materia orgánica (Walkley y Black), nitrógeno total (micro-Kjeldalhl), fósforo aprovechable (Bray P1), densidad aparente (probeta), textura (pipeta) y pH (potenciómetro). De una muestra compuesta de la capa arable $(0-20 \mathrm{~cm})$ de la parcela experimental se determinó: materia orgánica, nitrógeno total, fósforo aprovechable, $\mathrm{pH}$ y retención de fosfatos (método de Blakemore) (Ver tabla 1).

El suelo corresponde a un Andosol háplico con textura franco-limosa y densidad aparente (DAp) media a baja. La capa arable presenta alto contenido de materia orgánica y nitrógeno, y bajo en fósforo de acuerdo con

Tabla 1. Caracterización física y química del suelo

\begin{tabular}{|c|c|c|c|c|c|c|c|}
\hline Horizonte & $\begin{array}{c}\text { Profundidad } \\
(\mathbf{c m})\end{array}$ & DAp & Textura & $\begin{array}{c}\text { Porcen- } \\
\text { taje de } \\
\text { Materia } \\
\text { Orgánica }\end{array}$ & $\mathbf{p H}$ & $\begin{array}{c}\text { Porcenta- } \\
\text { je de Ni- } \\
\text { trógeno }\end{array}$ & $\begin{array}{c}\text { Fósforo } \\
(\mathbf{p p m})\end{array}$ \\
\hline $\mathrm{Ap}$ & $0-14$ & 1.1 & Franco limoso & $9.0 \%$ & 5.8 & $3.1 \%$ & 1.7 \\
\hline $\mathrm{A}_{2}$ & $14-29$ & 0.7 & Franco limoso & $3.6 \%$ & 5.9 & $1.8 \%$ & 1.9 \\
\hline $\mathrm{Bw}_{1}$ & $29-38$ & 0.8 & Franco limoso & $2.7 \%$ & 5.9 & $1.3 \%$ & 1.5 \\
\hline $\mathrm{Bw}_{2}$ & $38-48$ & 0.8 & Franco limoso & $1.7 \%$ & 5.9 & $0.8 \%$ & 0.4 \\
\hline $\mathrm{Bw}_{3}$ & $48-80$ & 0.8 & Franco limoso & $1.4 \%$ & 5.9 & $0.6 \%$ & 0.3 \\
\hline $\mathrm{Bw}_{4}$ & $80-89$ & 1.0 & Franco & $0.7 \%$ & 6.2 & $0.3 \%$ & 0.1 \\
\hline $2 \mathrm{C}$ & $89-114$ & 1.1 & Franco arenoso & $0.7 \%$ & 6.2 & $0.2 \%$ & 0.2 \\
\hline $\mathrm{MC}^{*}$ & $0-15$ & 1.1 & Franco limoso & $5.5 \%$ & 5.5 & $2.1 \%$ & 0.5 \\
\hline
\end{tabular}

${ }^{\star}$ Nota: MC: Muestra compuesta de la parcela 
Ortiz (2010) y la NOM-021; el pH es ligeramente ácido (5.8 a 6.2). La densidad aparente superior a $1 \mathrm{~g} . \mathrm{cm}^{-3}$ sugiere que el suelo tiene propiedades vítricas (IUSS Grupo de Trabajo WRB, 2007), y por la misma razón baja retención de fosfatos.

Se evaluaron los factores arreglo topológico, densidad de población, variedad de maíz y fertilización. El arreglo topológico consistió en franjas de maíz-frijol de guía asociados e intercalados con haba: a. 1x1 (un surco de maíz-frijol de guía asociado, intercalado con un surco de haba); y b. 2x2 (dos surcos de maíz-frijol de guía asociados, intercalados con dos surcos de haba).

La densidad de población, variedad de maíz y fertilización se eligieron a través de una matriz baconiana con un tratamiento adicional (nivel alto de densidad con maíz rosado) (Tabla 2) para observar diferencias en función de la densidad en dos variedades de maíz (Ver tabla 2).

Además, se establecieron 3 tratamientos de cultivo simple 1) maíz azul-frijol de guía asociados, 2) maíz rosado-frijol de guía asociados y 3) haba, para calcular la eficiencia relativa de la tierra $\left(E^{2} T^{3}\right)$ y la eficiencia relativa de la ganancia (ERG) (Ver tabla 3).

El diseño experimental fue en parcelas divididas con tres repeticiones en bloques al azar. Se consideró como parcela grande el arreglo topológico y como parcela chica los nueve tratamientos obtenidos de la matriz baconiana. Como unidad experimental seis surcos de $6 \mathrm{~m}$, de los que se consideraron como parcela útil $4 \mathrm{~m}$.

Tabla 2. Tratamientos según densidad de población - variedad del maíz y fertilización

\begin{tabular}{|c|c|c|c|c|c|c|c|c|c|}
\hline \multirow{4}{*}{ 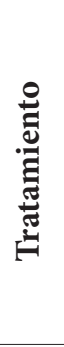 } & \multicolumn{6}{|c|}{ Maíz-frijol de guía } & \multicolumn{3}{|c|}{ Haba } \\
\hline & \multicolumn{2}{|c|}{$\begin{array}{c}\text { Densidad de población } \\
\text { (plantas ha-1) }\end{array}$} & \multirow{3}{*}{$\begin{array}{c}\text { Variedad } \\
\text { de maíz }\end{array}$} & \multicolumn{3}{|c|}{ Fertilización } & \multirow{3}{*}{$\begin{array}{c}\text { Densidad de } \\
\text { población } \\
\text { (plantas } \\
\text { ha }^{-1} \text { ) }\end{array}$} & \multicolumn{2}{|c|}{ Fertilización } \\
\hline & Maíz & Frijol & & $\mathbf{N}$ & $\mathrm{P}_{2} \mathrm{O}_{5}$ & Composta & & $\mathrm{P}_{2} \mathrm{O}_{5}$ & Composta \\
\hline & & & & & $\left.\mathrm{ha}^{-1}\right)$ & $\left(\mathbf{t ~ h a}^{-1}\right)$ & & $\left(\mathrm{kg} \mathrm{ha}^{-1}\right)$ & $\left(\mathrm{t} \mathrm{ha}^{-1}\right)$ \\
\hline $1^{\mathrm{z}}$ & 41,666 & 20,833 & Azul & 80 & 80 & 3 & 41,666 & 80 & 0 \\
\hline 2 & $29,166^{*}$ & $14,583^{*}$ & Azul & 80 & 80 & 3 & $29,166^{*}$ & 80 & 0 \\
\hline 3 & $41,666^{*}$ & $10,417^{\star}$ & Azul & 80 & 80 & 3 & 41,666 & 80 & 0 \\
\hline 4 & 41,666 & 20,833 & Azul & $0^{*}$ & $0^{*}$ & $\mathbf{0}$ & 41,666 & $0^{*}$ & $0^{*}$ \\
\hline 5 & 41,666 & 20,833 & Azul & $8^{*}$ & $80^{*}$ & $\mathbf{0}$ & 41,666 & $\mathbf{8 0}^{*}$ & $0^{*}$ \\
\hline 6 & 41,666 & 20,833 & Azul & $0^{*}$ & $\mathbf{0}^{*}$ & 3 & 41,666 & $0^{*}$ & $3^{*}$ \\
\hline 7 & $54,166^{*}$ & $27,083^{\star}$ & Azul & 80 & 80 & 3 & $54,166^{\star}$ & 80 & 0 \\
\hline 8 & 41,666 & 20,833 & Rosado & 80 & 80 & 3 & 41,666 & 80 & 0 \\
\hline 9 & $54,166^{\star}$ & $27,083^{\star}$ & Rosado & 80 & 80 & 3 & $54,166^{\star}$ & 80 & 3 \\
\hline
\end{tabular}

Nota: $\mathrm{z}$ - Tratamiento de referencia // ${ }^{\star}$ resaltan en negritas los niveles del factor estudiado.

3. Dentro del documento, el autor también se referirá a este término mediante su acrónimo.

Fertilización mineral y orgánica en el cultivo intercalado maíz-frijol asociados y haba en un Andosol del Estado de México 
Tabla 3. Tratamientos de cultivo simple

\begin{tabular}{|c|c|c|c|c|c|c|c|c|c|}
\hline \multirow{4}{*}{ 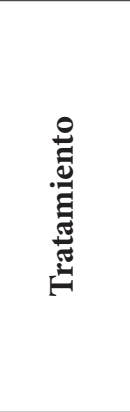 } & \multicolumn{6}{|c|}{ Maíz-frijol de guía } & \multicolumn{3}{|c|}{ Haba } \\
\hline & \multicolumn{2}{|c|}{$\begin{array}{c}\text { Densidad de población } \\
\left.\text { (plantas ha }{ }^{-1}\right)\end{array}$} & \multirow{3}{*}{$\begin{array}{l}\text { Variedad } \\
\text { de maíz }\end{array}$} & \multicolumn{3}{|c|}{ Fertilización } & \multirow{3}{*}{$\begin{array}{c}\text { Densidad } \\
\text { de pobla- } \\
\text { ción } \\
\text { (plantas } \\
\left.\text { ha }^{-1}\right)\end{array}$} & \multicolumn{2}{|c|}{ Fertilización } \\
\hline & Maíz & Frijol & & $\mathbf{N}$ & $\mathrm{P}_{2} \mathrm{O}_{5}$ & Composta & & $\mathbf{P}_{2} \mathbf{O}_{5}$ & Composta \\
\hline & & & & \multicolumn{2}{|c|}{$\left(\mathrm{kg} \mathrm{ha}^{-1}\right)$} & $\left(\mathrm{t} \mathrm{ha}^{-1}\right)$ & & $\left(\mathrm{kg} \mathrm{ha}^{-1}\right)$ & $\left(\mathbf{t ~ h a}^{-1}\right)$ \\
\hline CSMA & 41,666 & 20,833 & Azul & 80 & 80 & 3 & - & - & - \\
\hline CSMR & 41,666 & 20,833 & Rosado & 80 & 80 & 3 & - & - & - \\
\hline $\mathrm{CSH}$ & - & - & - & - & - & - & 41666 & 80 & 0 \\
\hline
\end{tabular}

Nota: ${ }^{\star} \mathrm{CSMA}=$ cultivo simple de maíz azul-frijol de guía // CSMR= cultivo simple de maíz rosado-frijol de guía // CSH=cultivo simple de haba.

Las variables en maíz estimadas fueron: rendimiento del grano con $14 \%$ de humedad, corrigiendo según la de humedad en campo, con un medidor de humedad de John Deere Moisture-Chek Tester modelo SW16060, y el desgrane. Para biomasa se consideró el peso seco del rastrojo más el peso del olote. En frijol se determinó el rendimiento de grano, y en haba el rendimiento de haba verde. Los rendimientos de los tres cultivos se reportan en una hectárea compacta, es decir, 0.5ha de la asociación maíz- frijol de guía y 0.5 ha de haba).

El análisis de varianza (ANOVA) se realizó de acuerdo con el diseño experimental de parce- las divididas con el paquete estadístico SAS (Statistical Análisis System) versión 9.1 (SAS Institute, 2004) y la separación de medias con la prueba de Diferencia Mínima Significativa (DMS) $(\mathrm{P}<0.05)$.

La ERT se realizó con base en Mead y Willey (1980) (Ver figura 1).

La eficiencia relativa de la ganancia se calculó de la misma manera que la ERT, pero con los ingresos brutos. Finalmente, para determinar el mejor tratamiento, se calculó el ingreso neto con costos fijos, variables y precio de venta obtenidos en la comunidad.

\begin{tabular}{|c|c|c|c|c|}
\hline \multirow{2}{*}{ ETR } & \multirow{2}{*}{$=$} & $\begin{array}{l}\text { Rend. maíz + rend. de frijol en el } \\
\text { sistema intercalado }\end{array}$ & \multirow{2}{*}{+} & Rend. haba en el sistema intercalado \\
\hline & & $\begin{array}{l}\text { Rend. maíz + rend. frijol como } \\
\text { cultivo simple }\end{array}$ & & Rend. haba como cultivo simple \\
\hline
\end{tabular}

Figura 1. Fórmula Mead y Willey. 


\section{Resultados y Discusión}

\section{Rendimiento del cultivo intercalado de maíz-frijol de guía asociados y haba}

En maíz y frijol, el rendimiento no se vio afectado por el arreglo topológico (parcela grande), pero sí por la parcela chica. En el haba la respuesta fue a la inversa.

En rendimiento de grano y rastrojo de maíz se encontraron diferencias significativas, debido a la densidad de población, la cual ocasionó un incremento en el rendimiento de grano de $53 \%$ y de $66 \%$ en rastrojo. Según Rojas et al. (2017), al intensificarse el sistema, existe un mejor aprovechamiento de los recursos suelo, luz y agua y también se informa que el rendimiento de maíz intercalado con quinchocho (Cajanus cajan) con y sin fertilización, no mostró diferencias según (Quiroz y Marín 2003); y que el rastrojo tendió a aumentar tiende a incrementarse con aumentos en la densidad de plantas (De la Cruz et al. 2009).

La fertilización mineral, organo-mineral y orgánica incrementaron significativamente el rendimiento de grano de maíz (tabla 4). En el rastrojo, solo la fertilización mineral mostró efecto, debido según Brady y Weil (1999) a que la producción de rastrojo depende más del contenido de nutrientes disponibles en los fertilizantes minerales y menos de los disponibles de liberación lenta ligados al abono orgánico (Ver tabla 4).

\section{Rendimiento de frijol de guía}

El rendimiento del frijol en el arreglo topológico $1 \mathrm{x} 1$ no fue diferente del 2x2. El frijol es una planta con metabolismo C3 y muestra plasticidad para adaptarse a las diferentes condiciones de entrada de luz en los arreglos estudiados (Escalante y Kohashi, 1993).

Al aumentar la densidad, el rendimiento de grano incrementó $48 \%$ por lo que se considera que aún se puede incrementar la densidad del frijol. La fertilización no presentó diferencias, lo que muestra que el suelo está proporcionando los nutrientes necesarios para este cultivo (Escalante y Kohashi, 1993) o está haciendo uso de los nutrientes suministrados al maíz.

Tabla 4. Rendimiento del grano y rastrojo del maíz azul asociado con frijol de guía e intercalado con haba en función de la dosis y fuente de fertilización

\begin{tabular}{|c|c|c|}
\hline Dosis & $\begin{array}{c}\text { Rendimiento de grano } \\
\left(\mathbf{k g ~ 0 . 5} \mathbf{~ h a}^{-1}\right)\end{array}$ & $\begin{array}{c}\text { Rendimiento de rastrojo } \\
\left(\mathbf{k g ~ 0 . 5} \mathbf{~ h a}^{\mathbf{1})}\right.\end{array}$ \\
\hline $00-00-00-0 \mathrm{C}$ & $867.0 \mathrm{~b}^{*}$ & $2.6 \mathrm{~b}$ \\
\hline $00-00-00-3 \mathrm{C}$ & $1799.1 \mathrm{a}$ & $2.7 \mathrm{~b}$ \\
\hline $80-80-00-0 \mathrm{C}$ & $1541.4 \mathrm{a}$ & $3.7 \mathrm{a}$ \\
\hline $80-80-00-3 \mathrm{C}$ & $1422.5 \mathrm{a}$ & $3.9 \mathrm{a}$ \\
\hline DMS & 536.5 & 0.9 \\
\hline
\end{tabular}

Nota: ${ }^{\star}$ Letras diferentes en columnas indican diferencias significativas según la prueba de DMS $\alpha=0.05$. 


\section{Rendimiento de haba verde}

El rendimiento de haba verde fue $18 \%$ más alto en el arreglo 1x1. Esto muestra competencia por recursos más intensa entre el haba con su misma especie que con el maíz. Rezaei-Chianeh et al. (2011) han reportado competencia intraespecífica e interespecífica cuando se siembra maíz y haba asociados. Tampoco se observó respuesta a la densidad de plantas, lo cual difiere a lo reportado por otros estudios (López-Bellido y López-Bellido, 2003; Rojas et al., 2012).

La fertilización tampoco afectó el rendimiento de haba verde, lo cual pudo deberse a que las dosis aplicadas no satisficieron la demanda del cultivo. Pichardo et al. (2007) mencionan que la aplicación fraccionada de 130 unidades de $\mathrm{N}$, superior a la dosis en este estudio, incrementó el rendimiento. Estos resultados también difieren de lo encontrado por Morales et al. (2002) y Escalante y Rodríguez (2011), que mencionan que la fertilización con fósforo tiene un efecto positivo en la producción de haba cuando se acompaña con la aplicación de nitrógeno; sin embargo, los primeros mencionan que la variedad criolla no respondió a la aplicación de fósforo lo que concuerda con este estudio.

\section{Eficiencia relativa de la tierra (ERT)}

La eficiencia relativa de la tierra en los dos arreglos topológicos varió de 1.17 a 1.40 (ta- bla 5). Ligarreto et al. (1992), obtuvieron ERT de 1.61 y 1.54 para proporciones de plantas de maíz y fríjol de 4:2 y 4:1. Quiroz y Marín (2003), obtuvieron valores de 1.90 y 1.93 de ERT en el sistema intercalado de maíz y quinchoncho (C. cajan); y Morales et al. (2006), obtuvieron ventajas de 60 a $200 \%$ en girasol Victoria con diferentes cultivares de frijol.

El mayor aporte a la ERT total en este estudio lo hizo el cultivo de haba cuya ERT parcial varió de 0.75 a 0.90 . No obstante, como el maíz es el cultivo de mayor prioridad por ser el alimento básico, los mejores tratamientos serían el 1x1 en caso de maíz azul y el 2x2 el caso de maíz rosado por la ERT parcial en dichos tratamientos de 0.50 y 0.60 (Ver tabla 5).

\section{Eficiencia relativa de la ganancia (ERG)}

La eficiencia relativa de la ganancia $(E R G)^{4}$ en los dos arreglos topológicos fue mayor a 1 , variando de 1.21 a 1.44. El haba dio el mayor aporte según las ERG parciales, por los rendimientos y su mayor valor en el mercado. Albino-Garduño (2014) obtuvo una ERG de 1.58 en el cultivo intercalado de maíz y frijol de mata con el arreglo topológico $1 x 1$.

\section{Ingreso neto}

El ingreso neto en términos de salarios mínimos dia $^{-1}$ al año varió de 1.79 a 2.40 (Cuadro 6) y los mejores tratamientos el arreglo topológico $1 x 1$ de maíz azul y el arreglo $2 \times 2$ de maíz rosado (Ver tabla 6 ).

4. Dentro del documento, los autores también se referirán a este término mediante su acrónimo. 
Tabla 5. Efecto de la variedad de maíz de la eficiencia relativa de la tierra (ERT) del cultivo maíz-frijol de guía asociados intercalado con haba, bajo dos arreglos topológicos

\begin{tabular}{|c|c|c|c|c|c|}
\hline \multirow{3}{*}{ Cultivo intercalado } & \multicolumn{2}{|c|}{ Rendimiento } & \multicolumn{2}{|c|}{ ETR parciales } & \multirow{3}{*}{ ETR Tota } \\
\hline & Maíz + Frijol & Haba verde & \multirow{2}{*}{ Maíz + Frijol } & \multirow{2}{*}{ Haba verde } & \\
\hline & \multicolumn{2}{|c|}{$\left(\mathrm{t} 0.5 \mathrm{ha}^{-1}\right)$} & & & \\
\hline $\begin{array}{l}\text { 1x1 maíz azul-frijol de guía, } \\
\text { intercalado con haba }\end{array}$ & $\begin{array}{c}(1.6+0.144) \\
1.744\end{array}$ & 6.30 & 0.50 & 0.90 & 1.40 \\
\hline $\begin{array}{l}\text { 1x1 maíz rosado-frijol de } \\
\text { guía, intercalado con haba }\end{array}$ & $\begin{array}{c}(1.234+0.083) \\
1.317\end{array}$ & 5.40 & 0.40 & 0.77 & 1.17 \\
\hline $\begin{array}{l}\text { 2×2 maíz azul-frijol de guía } \\
\text { intercalado con haba }\end{array}$ & $\begin{array}{c}(1.3+0.145) \\
1.445\end{array}$ & 5.90 & 0.40 & 0.84 & 1.24 \\
\hline $\begin{array}{l}\text { 2x2 maíz rosado-frijol de } \\
\text { guía, intercalado con haba }\end{array}$ & $\begin{array}{c}(1.725+0.098) \\
1.823\end{array}$ & 5.30 & 0.60 & 0.75 & 1.35 \\
\hline \multirow{2}{*}{ Cultivo simple } & Maíz + Frijol & Haba verde & & & \\
\hline & \multicolumn{2}{|c|}{$\left(\mathrm{tha}^{-1}\right)$} & & & \\
\hline Maíz azul-frijol de guía & $\begin{array}{c}(3.275+0.219) \\
3.494\end{array}$ & & & & \\
\hline Maíz rosado-frijol de guía & $\begin{array}{c}(3.141+0.135) \\
3.276\end{array}$ & & & & \\
\hline Haba & & 7.0 & & & \\
\hline
\end{tabular}

Ruiz y Loaeza (2004) mencionan que sembrar surcos alternos asegura el ingreso neto del productor; en su estudio, el cultivo de mayor valor económico fue el frijol con mayor probabilidad de producir sin ocupar una mayor proporción de terreno.

\section{Conclusiones}

En la evaluación del sistema de milpa integrada por el cultivo de maíz-frijol intercalado con haba, según arreglo topológico, densidad, variedad de maíz y fertilización se concluye que, la incorporación de abonos orgánicos como fertilización presenta ventajas importantes, mayores combinada con la fertilización mineral; con variación en los diferentes cultivos.

El cultivo intercalado supera al cultivo simple: es mejor sembrar el maíz-frijol intercalado con haba y se continúa produciendo maíz y frijol, que son la base de la alimentación de los sistemas campesinos. Por lo tanto, la recomendación para el productor sería sembrar bajo el arreglo topológico 1x1 de maíz azul o $2 \times 2$ de maíz rosado por los valores más altos de la ERT y la ERG e ingreso neto. 
Tabla 6. Eficiencia relativa de la ganancia (ERG), ingreso neto y salario mínimo por día

\begin{tabular}{|c|c|c|c|c|c|c|c|}
\hline \multirow[b]{2}{*}{ Cultivo intercalado } & \multirow[b]{2}{*}{$\begin{array}{c}\text { Maíz +Frijol } \\
(\$)\end{array}$} & \multirow[b]{2}{*}{$\begin{array}{l}\text { Haba } \\
\text { verde } \\
(\$)\end{array}$} & \multicolumn{2}{|c|}{ ERG parciales } & \multirow[b]{2}{*}{ ERG } & \multirow[b]{2}{*}{$\begin{array}{l}\text { Ingreso } \\
\text { neto }\end{array}$} & \multirow[b]{2}{*}{$\begin{array}{l}\text { Salario } \\
\text { mínimo } \\
\text { por día }\end{array}$} \\
\hline & & & $\begin{array}{c}\text { Maíz } \\
+ \text { Frijol }\end{array}$ & $\begin{array}{l}\text { Haba } \\
\text { verde }\end{array}$ & & & \\
\hline $\begin{array}{l}\text { 1x1 maíz azul-frijol guía } \\
\text { intercalado con haba }\end{array}$ & $\begin{array}{c}(5,600+3,312) \\
8,912\end{array}$ & 63,000 & 0.54 & 0.90 & 1.44 & 61,222 & 2.4 \\
\hline $\begin{array}{c}\text { 1x1 maíz rosado-frijol } \\
\text { de guía, intercalado con } \\
\text { haba }\end{array}$ & $\begin{array}{c}(4,319+1,909) \\
6,228\end{array}$ & 54,000 & 0.44 & 0.77 & 1.21 & 45,142 & 1.79 \\
\hline $\begin{array}{l}\text { 2x2 maíz azul-frijol de } \\
\text { guía, intercalado con haba }\end{array}$ & $\begin{array}{c}(4,550+3,335) \\
7,885 \\
\end{array}$ & 59,000 & 0.48 & 0.84 & 1.32 & 43,855 & 1.71 \\
\hline $\begin{array}{c}\text { 2x2 maíz rosado con fri- } \\
\text { jol de guía, intercalado } \\
\text { con haba }\end{array}$ & $\begin{array}{c}(6,037+2,254) \\
8,291\end{array}$ & 53,000 & 0.59 & 0.76 & 1.35 & 46,962 & 1.8 \\
\hline Cultivo simple & $\begin{array}{c}\text { Maíz +Frijol } \\
(\$)\end{array}$ & $\begin{array}{l}\text { Haba } \\
\text { verde }\end{array}$ & & & & & \\
\hline Maíz azul-frijol de guía & $\begin{array}{c}(11,462+5,037) \\
16499\end{array}$ & 0 & & & & 11,911 & 0.46 \\
\hline Maíz rosado-frijol de guía & $\begin{array}{c}(10,993+3,105) \\
14098\end{array}$ & 0 & & & & 8,310 & 0.32 \\
\hline Haba & & 70,000 & & & & 49,459 & 1.9 \\
\hline
\end{tabular}

\section{Referencias}

Albino-Garduño, R. (2014). El Sistema Agroforestal Milpa Intercalada con Árboles Frutales (MIAF), productividad y optimización económica del maíz y frijol. (Tesis de Doctorado). Colegio de Postgraduados. Montecillo, México

Albino-Garduño, R.; Turrent-Fernández, A.; Cortés-Flores, J.; Livera-Muñoz, M. y MendozaCastillo, M. (2015). Distribución de raíces y de radiación solar en el dosel de maíz y frijol intercalado. Agrociencia, 49 (5), 513-531

Alcalá J, M.; Ortiz S., C.A. y Gutiérrez C., M.C. (2001). Clasificación de los suelos de la Meseta Tarasca, Michoacán. Terra Latinoamericana 19(3), 227-239

Brady, N.C., y Weil, R.R. (1999). The nature and properties of soils. New Jersey, USA: Prentice Hall 
Cuanalo C., H. (1990). Manual para la descripción de perfiles de suelos en el campo. Chapingo, México: Colegio de Posgraduados

De la Cruz L., E.; Córdova O., H.; Estrada B., M.A.; Mendoza P., J.D.; Gómez V., A. y Brito M., N.P. (2009). Rendimiento de grano de genotipos de maíz sembrados bajo tres densidades de población. Revista Universidad y Ciencia 25(1), 93-98

Escalante E., J.A. y Rodríguez G., M.T. (2011). Biomasa y rendimiento en haba en función de la densidad de población, nitrógeno y fosforo. Revista Ciencias Agrícolas Informa 20(1),16-25

Escalante E., J.A., y Kohashi, S. (1993). El rendimiento y crecimiento del frijol. Manual para la toma de datos. Montecillo, México: Colegio de Posgraduados

García O., J.A.; Cedillo G., J.G.; Juan P., J.I. y Balderas P., M.A. (2012). Procesos de cambio en el uso del suelo de una microcuenca en el altiplano mexicano: el caso del río San José en el Estado de México. Papeles de geografía 55-56, 63-73

Guerrero M., S. (1987). Fertilización de maíz (Zea mays L.) con porqueraza y su efecto residual en un andisol de la Sierra Purépecha. (Tesis de maestría). Colegio de Postgraduados. Chapingo, México

IUSS Grupo de Trabajo WRB. (2007). Base Referencial Mundial del Recurso Suelo. Informes sobre Recursos Mundiales de Suelos. Roma, Italia: FAO

IUSS Grupo de Trabajo WRB. (2014). Base Referencial Mundial del Recurso Suelo. Informes sobre Recursos Mundiales de Suelos. Roma, Italia: FAO

Ligarreto, G.; Bastida, G. y Castillo, M. (1992). Efecto de las densidades de siembra en el rendimiento de la asociación maíz-frijol voluble. Revista ICA, 27, 25-33

López-Bellido G., R.J. y López-Bellido G., F.J. (2003). La densidad de plantas en el cultivo de las habas. Agricultura: Revista agropecuaria (849):196-199

Mead, R. y Willey W.R. (1980). The concept of a "Land Equivalent Ratio" and advantages in yields from intercropping. Experimental Agriculture, 16(3), 217-228 
Mendieta F., A.M; Guerrero P., A.G y Estrada F., J.G. (2013). Propuesta de manejo de un sistema agroforestal en la zona de amortiguamiento de la Reserva de la Biosfera Mariposa Monarca, Ejido el Depósito, San José del Rincón, Estado de México. En: Álvarez, G.F., Ortiz T., E., Bahena J., F., Pérez M., A., Carranza C., I., Pérez R., E., Díaz R., R., Villanueva J., J.A., Ocampo F., I. y Villareal M., L.A. (Eds.). Agricultura Sostenible Vol. 9 (pp. 121-137). Puebla, México: Sociedad Mexicana de Agricultura Sostenible

Morales R., E.J.; De la O A., H.; Morales R., A. y De la Cruz A., V.M. (2002). Evaluación de cinco genotipos de haba (Vicia faba L.) con seis niveles de fósforo en Tecámac, México. Ciencia Ergo Sum 9(2), 183-189

Morales R., E.J.; Escalante E., J.A.; Tijerina C., L.; Volke H., V. y Sosa M., E. (2006). Biomasa, rendimiento, eficiencia en el uso del agua y de la radiación solar del agrosistema girasolfrijol. Terra Latinoamericana 24(1), 55-64

Norma Oficial Mexicana NOM-021-SEMARNAT (2000), Que establece las especificaciones de fertilidad, salinidad y clasificación de suelos. Estudios, muestreo y análisis. Recuperado de http://www.semarnat.gob.mx/leyes-y-normas/nom-suelos

Núñez S., A.E.; Carrión, G.; Núñez C., M.C. y Rebolledo D., O. (2003). Densidad de población de Quistes de Globodera rostochiensis Woll. en el Cofre de Perote, Veracruz, México. Revista Mexicana de Fitopatología, 21(2), 207-213

Ortiz S., C.A. (2010). Edafología. Chapingo. México: Universidad Autónoma Chapingo

Pichardo-Riego, J.C.; Escalante-Estrada, J.A.; Rodríguez-González, M.T. y Sánchez-García, P. (2007). Aplicación dividida y eficiencia agronómica de nitrógeno, uso de agua y radiación, y rendimiento de haba. Terra Latinoamericana, 25(2), 145-154

Quiroz A., I. y Marín, D. (2003). Rendimiento en granos y eficiencia de una asociación maíz (Zea mays) y quinchoncho (Cajanus cajan) con o sin fertilización. Revista Bioagro, 15(2), 121-128

Rezaei-Chianeh, E.; Nassab A.D., M.; Shakiba, M.R.; Ghassemi-Golezani, K.; Aharizad, S. y Shekari, F. (2011). Intercropping of maize (Zea mays L) and faba bean (Vicia faba L) at different plant population densities. African Journal of Agricultural Research, 6(7), 1786-1793 
Rojas T., J.; Díaz R., R.; Álvarez G., F.; Ocampo M., J. y Escalante E., J.A. (2012). Tecnología de producción de haba y características socioeconómicas de productores en Puebla y Tlaxcala. Revista mexicana de ciencias agrícolas, 3(1), 35-49

Rojas V., N.J.; Escalante E., J.A.S.; Conde M., F.V.; Mejía C., J.A. y Díaz R., R. (2017). Rendimiento del frijol ayocote y maíz del agrosistema asociado en función del número de plantas por mata. Terra Latinoamericana, 35(3), 219-228

Ruiz V., J. y Loaeza R., G. (2004). Validación del método de siembra en surcos alternos para la asociación maíz-frijol en Valles Centrales de Oaxaca. Naturaleza y Desarrollo, 2(1), 13-17 SAS, Institute (2004). SAS/STAT ${ }^{\oplus}$ Versión 9.1. SAS Institute Inc. Cary, NC, USA SEMARNAT - COLPOS (2002). Inventario Nacional de Suelo. Degradación del suelo causada por el hombre 1:200,000

SIAP (2013). Cierre de la producción agrícola por cultivo. Recuperado de: www.siap.gob.mx/ cierre-de-la-produccion-agricola-por-cultivo/

SIAP (2017). Cierre de la producción agrícola por cultivo. Recuperado de: http://nube.siap.gob. $\mathrm{mx} /$ cierreagricola/

Van, R. L.P. (1999). Procedimientos para análisis de suelos. (Traductores: Gutiérrez C., M.C., Tavares E., C.A. y Ortiz S., C.A.). Montecillo, México: Colegio de Postgraduados 